\title{
Model Predictive Path-Following for Constrained Nonlinear Systems
}

\author{
T. Faulwasser, B. Kern and R. Findeisen
}

\begin{abstract}
We propose a model predictive control approach to path-following problems of constrained nonlinear systems. We directly consider input and state constraints. Furthermore, we introduce an extended corridor path-following problem, which allows to add spatial degrees of freedom to the path formulation. We give sufficient stability conditions for predictive solutions to $1 \mathrm{~d}$ and corridor path-following problems. To illustrate the performance of our approach we discuss the example of an autonomous vehicle subject to input constraints.
\end{abstract}

Index Terms - Nonlinear model predictive control, corridor path-following, input and state constraints, stability.

\section{INTRODUCTION}

Classical controller design either aims at setpoint stabilization or intends to track dynamically varying reference signals and trajectories. Recently, path-following has been proposed to provide an alternative problem formulation to trajectory tracking [1], [2]. While in tracking problems the reference trajectory defines when the system should be where in the state space the main idea of path-following is different: Design a controller, that affects both the system as well as the timing when to be where on a reference path. And additionally, it should guarantee that the system moves as close as possible to and along the path through the state space. In fact, path-following provides a suitable framework for different problem formulations: Ship or flight course control, the car-parking problem or the control of robots and CNC-machines [4], [13]. It is also appropriate for control problems arising in batch crystallization [11].

Existing path-following approaches are limited, since input constraints are not explicitly considered [1], [2], [13]. In this contribution we propose a solution to the path-following problem for nonlinear systems subject to constraints on inputs and states. In order to consider constraints the proposed control scheme relies on nonlinear model predictive control (NMPC) [6], [8], [9]. Since NMPC can be applied to tracking problems (see [10] for early results) we point out the difference between tracking and path-following in detail in Section II. As mentioned, to follow a parametrized reference path through the state space means to design a control scheme which affects both - the systems behavior and the evolution of the (parametrized) reference. This is related to the reference governor approach, which has been previously discussed in the context of predictive control, see

T. Faulwasser, B. Kern and R. Findeisen are with the Institute for Automation Engineering, Otto-von-Guericke University Magdeburg, Germany. T. Faulwasser and B. Kern are member of the International Max Planck Research School for Analysis, Design and Optimization in Chemical and Biochemical Process Engineering Magdeburg. \{timm. faulwasser, benjamin.kern, rolf.findeisen\}@ovgu.de e.g. [3], [12]. Mainly, these results rely on two control loops: An inner one, which affects the system directly and an outer one, which controls the reference evolution. In contrast, we propose a method that relies on one control loop.

In Section III we present our main result: An NMPC approach to solve the path-following problem for nonlinear systems subject to input and state constraints while guaranteeing stability. The presented results extend those given in [5]. Mainly, we show that the proposed nonlinear model predictive path-following control (MPFC), which regards the path parameter as an additional state variable, provides a suitable control setup for the path-following problem. We also give sufficient stability conditions. While other works on path-following consider output-feedback [1], [2], [13], we rely on state feedback. In Section IV we illustrate our scheme by simulations of an autonomous vehicle. In Section V we extend our scheme to consider spatial degrees of freedom in the path definition and the controller design.

\section{PATH-FOLLOWING AND TRACKING}

Consider a continuous time, nonlinear system of the form

$$
\dot{x}(t)=f(x(t), u(t)), \quad x\left(t_{0}\right)=x_{0},
$$

where $x \in \mathcal{X} \subseteq R^{n}$ and $u \in \mathcal{U} \subseteq R^{m}$ define state and input constraints. The objective of trajectory tracking is to design a controller, such that the system state $x(t)$ tracks a time-dependent reference signal $r(t)$. Often, the reference trajectory $r(t)$ is assumed to be generated by a known or unknown exo-system. By defining the error variable

$$
e_{t}(t):=x(t)-r(t)
$$

the tracking problem can be reformulated as a setpoint stabilization problem: $\lim _{t \rightarrow \infty} e_{t}(t)=0$.

Path-following refers to a different control problem. Instead of tracking a time-dependent reference trajectory $r(t)$ a parametrized reference path has to be followed. This path is given by a regular curve $\mathcal{P} \subset \mathcal{X}$ in the state space $\mathbb{R}^{n}$

$$
\mathcal{P}=\left\{p(\theta) \in \mathbb{R}^{n}: \theta \in[\hat{\theta}, 0] \mapsto p(\theta)\right\} .
$$

The path $\mathcal{P}$ is specified by the map $p:[\hat{\theta}, 0] \mapsto \mathbb{R}^{n}$, which projects the negative real interval $[\hat{\theta}, 0]$ to the state space $\mathbb{R}^{n}$. Note, that the path parameter $\theta \leq 0$ and thus the path $\mathcal{P}$ is called negatively parametrized. It ends in the origin $p(\theta=$ $0)=0$. Additionally, we assume the map $p$ to be bijective and sufficiently often continuously differentiable. Using the path definition (3), the path-following error is defined:

$$
e_{p}(t):=x(t)-p(\theta(t)) .
$$


The control objective is to guarantee that the path-following error converges $\lim _{t \rightarrow \infty} e_{p}(t)=0$.

As mentioned the reference signal for tracking problems $r(t)$ is directly time dependent and explicitly specifies when to be where in the state space. In path-following the time plays a secondary role. Although the path parameter $\theta(t)$ is in general time-dependent, its time evolution $\dot{\theta}$ is not given a priori. Rather, it is obtained in the controller to achieve forward movement and error minimization. While in tracking a specific reference trajectory $r(t)$ has to be tracked in the time domain, in path-following the objective is to follow one $p(\theta(t)) \in \mathcal{P}$ as good as possible. In the remain of this work we consider the following control problem.

\section{Path-Following Problem}

Design a controller to satisfy the following:

P1 Path convergence: The path-following error vanishes asymptotically $\lim _{t \rightarrow \infty} e_{p}(t)=0$.

P2 Forward motion: $\dot{\theta}(t)>0$ holds for all $\theta(t) \in[\hat{\theta}, 0)$.

P3 Constraint satisfaction: The state and input constraints $x(t) \in \mathcal{X} \subseteq \mathbb{R}^{n}$ and $u(t) \in \mathcal{U} \subset \mathbb{R}^{m}$ are respected for all $t \geq 0$.

\section{MODEL PREDICTIVE PATH-FOLLOWING}

Subsequently, we propose a scheme for nonlinear model predictive path-following control to solve the considered problem. Our main idea is: Treat the path parameter as an additional state variable in an expanded predictive setup and obtain its evolution and the real system input by solving an open-loop optimal control problem.

To solve the path-following problem a sampled data NMPC strategy is considered. Predicted system states and inputs are denoted by $\bar{x}(\cdot)$ and $\bar{u}(\cdot)$. In contrast to standard NMPC approaches (see e.g. [6], [8], [9]) the cost functional to be minimized at all sampling instances $t_{k}=k \delta$ is given by

$$
\begin{array}{rl}
J(\bar{x}, \bar{u}, \theta, v)=\int_{t_{k}}^{t_{k}+T_{P}} & F(\bar{x}, \bar{u}, \theta, v) d \tau \\
& +E\left(\bar{x}\left(t_{k}+T_{P}\right), \theta\left(t_{k}+T_{P}\right)\right)
\end{array}
$$

where $F(\cdot)$ denotes the cost function and $E(\cdot)$ is the end penalty. Besides $\bar{x}$ and $\bar{u}$ the cost functional depends on the path parameter $\theta$ and a not yet defined path parameter input $v$. The proposed control strategy is the repeated solution of the open-loop optimal control problem ${ }^{1}$

$$
\underset{\bar{u}(\cdot), v(\cdot)}{\operatorname{minimize}} J(\bar{x}, \bar{u}, \theta, v)
$$

which is subject to the system model and the constraints

$$
\begin{aligned}
& \dot{\bar{x}}(\tau)=f(\bar{x}(\tau), \bar{u}(\tau)), \quad \bar{x}\left(t_{k}\right)=x\left(t_{k}\right), \\
& \forall \tau \in\left[t_{k}, t_{k}+T_{P}\right]: \quad \bar{x}(\tau) \in \mathcal{X}, \bar{u}(\tau) \in \mathcal{U}, \\
& \bar{x}\left(t_{k}+T_{P}\right) \in \mathcal{E} \subseteq \mathcal{X} \subseteq \mathbb{R}^{n} .
\end{aligned}
$$

\footnotetext{
${ }^{1}$ Assuming for simplicity that the minimum is actually attained.
}

The terminal region $\mathcal{E}$ restricts the predicted state $\bar{x}(\cdot)$ to a specific region at the end of each prediction, i.e. at time $t_{k}+T_{p}$ the state needs to be in the set $\mathcal{E}$.

Besides these somehow standard constraints additional path-following constraints have to be respected:

$$
\begin{aligned}
& \dot{\theta}(\tau)=g(\theta(\tau), v(\tau)), \quad \theta\left(t_{k}\right)=\theta\left(t_{k} \mid \theta\left(t_{k-1}\right)\right), \\
& \forall \tau \in\left[t_{k}, t_{k}+T_{P}\right]: \theta(\tau) \in[\hat{\theta}, 0], v(\tau) \in \mathcal{V} .
\end{aligned}
$$

These extra constraints describe the evolution of the path, which is specified by the timing law $\dot{\theta}=g(\theta, v)$. The virtual input $v$ controls the evolution of $\theta$. Please note, that the timing law $g(\cdot)$ is usually not given a priori. Actually, it is an additional degree of freedom in the controller design. To solve the ODE (8a) an initial condition $\theta\left(t_{k}\right)$ is necessary at every sampling instance $t_{k}$. If an initial path point $p_{0}=$ $p\left(\theta_{0}\right)$ is given a priori, then the corresponding value of the path parameter $\theta_{0}$ serves as an initial condition for the first sampling instant. If no starting point $p_{0}$ is given, a path point close to $x_{0}$ should be calculated by (locally) solving the minimum distance problem minimize $\left\|x_{0}-p(\theta)\right\|$. At all following sampling instances the corresponding value of the previous one is used $\theta\left(t_{k}\right)=\theta\left(t_{k} \mid \theta\left(t_{k-1}\right)\right)$. The solution of (5)-(8) leads to the optimal input trajectory $u^{\star}\left(t \mid x\left(t_{k}\right)\right)$ which is applied during the time interval $t \in\left[t_{k}, t_{k}+\delta\right]$.

As mentioned before the proposed MPFC scheme given by (5) - (8) is an extended NMPC scheme. The open-loop optimal control problem is expanded by the virtual state $\theta$ and by the virtual input $v$. Essentially, $v$ controls the path parameter evolution $\dot{\theta}$. In general, the proposed approach will not lead to a time-optimal path evolution. Actually, considering the MPFC scheme path convergence is more important than speed.

\section{STABILITY OF MPFC}

In the following, we present sufficient stability conditions for the proposed expanded NMPC scheme. The assumptions follow well-known stability results, see e.g. [6], [8], [9]:

A1 The state constraint set $\mathcal{X} \subseteq \mathbb{R}^{n}$ is closed and connected and contains the origin in its interior.

A2 $\mathcal{U} \subseteq \mathbb{R}^{m}$ is compact and the origin is contained in the interior of $\mathcal{U}$.

A3 $f: \mathbb{R}^{n} \times \mathbb{R}^{m} \mapsto \mathbb{R}^{n}$ from (1) is a continuous and locally Lipschitz vector field. Furthermore, $f(0,0)=0$.

A4 For all initial conditions in the region of interest and any piecewise continuous input function $u(\cdot):\left[0, T_{P}\right] \mapsto \mathcal{U}$, (1) has a unique continuous solution.

A5 The cost function $F: \mathcal{X} \times \mathcal{U} \times[\hat{\theta}, 0] \times \mathcal{V} \mapsto \mathbb{R}$ is continuous and positive definite in the domain $\mathcal{X} \times \mathcal{U} \times$ $[\hat{\theta}, 0] \times \mathcal{V}$.

To ensure stability of the proposed MPFC scheme we additionally assume:

A6 The path $\mathcal{P}$ is given by a regular, sufficiently often differentiable, negatively parametrized and bijective map $p$, such that $\forall \theta \in[\hat{\theta}, 0]: \theta \mapsto p(\theta) \in \mathcal{X} \subseteq \mathbb{R}^{n}$ and $p(0)=0$ hold. 
Preprint of accepted submission to 48th IEEE Conference on Decision and Control '09, Shanghai, China.

A7 The timing law is chosen such that $g(\theta, v)$ has equivalent properties as required for $f(x, u)$ in assumptions $\mathrm{A} 3$ and A4. Furthermore, $\forall v \in \mathcal{V}$ and $\forall \theta \in[\hat{\theta}, 0)$ : $g(\theta, v)>0$, where $\mathcal{V} \subseteq \mathbb{R}$ is compact and contains 0 in its interior.

If these assumptions are fulfilled, then the following holds.

\section{Theorem 1 (Stability of MPFC)}

Consider the path-following problem for (1) as given by P1$P 3$ and assume that assumptions A1-A7 are fulfilled. Suppose that:

(i) The terminal region $\mathcal{E}$ is compact and $\mathcal{P} \subseteq \mathcal{E}$. The terminal penalty $E(x, \theta)$ is continuously differentiable, positive semidefinite and $E(0,0)=0$ holds.

(ii) For all $(x, \theta) \in \mathcal{E} \times[\hat{\theta}, 0]$ there exists a pair of admissible inputs $\left(u_{\mathcal{E}}, v_{\mathcal{E}}\right) \in \mathcal{U} \times \mathcal{V}$ such that

$$
\nabla E(x, \theta) \cdot\left(\begin{array}{l}
f\left(x, u_{\mathcal{E}}\right) \\
g\left(\theta, v_{\mathcal{E}}\right)
\end{array}\right)+F\left(x, \theta, u_{\mathcal{E}}, v_{\mathcal{E}}\right) \leq 0
$$

and the solutions of $\dot{x}=f\left(x, u_{\mathcal{E}}\right)$ and $\dot{\theta}=g\left(\theta, v_{\mathcal{E}}\right)$ starting at $(x, \theta) \in \mathcal{E} \times[\hat{\theta}, 0]$ stay in $\mathcal{E} \times[\hat{\theta}, 0]$ for all times.

(iii) The NMPC open-loop optimal control problem is feasible for $t_{0}$.

Then, for the closed-loop system defined by (1), (5)-(8), the path-following error $e_{p}(t)=x(t)-p(\theta(t))$ converges to zero for $t \rightarrow \infty$. Furthermore, the region of attraction is given by the set of states for which the open-loop optimal control problem (5)-(8) has a feasible solution.

Proof: The proof of Theorem 1 consists of two steps. First, we show that the proposed MPFC scheme is equivalent to setpoint stabilization in different coordinates. In these coordinates we apply well-known stability results for NMPC. Second, we demonstrate how the stability conditions map back to the original coordinates.

Step 1: Consider the change of coordinates

$$
\mathbf{T}:\left(\begin{array}{l}
x \\
\theta
\end{array}\right) \mapsto y=\left(\begin{array}{c}
x-p(\theta) \\
\theta
\end{array}\right), \quad\left(\begin{array}{l}
u \\
v
\end{array}\right) \mapsto w=\left(\begin{array}{l}
u \\
v
\end{array}\right) .
$$

In the new coordinates $y, w$ the control scheme (5)-(8) is equivalent to a NMPC setpoint stabilization in $\mathbb{R}^{n+1}$, thus we apply NMPC stability conditions directly, see e.g. [8], [9]. These are in fact equivalent to the conditions (i) and (iii).

In $y, w$ coordinates the augmented system dynamics are $\dot{y}=\tilde{f}(y, w)=\left(\dot{x}-\frac{\partial p}{\partial \theta} \dot{\theta}, \dot{\theta}\right)^{T}$. The terminal region in $y, w$ coordinates is the image of $\mathcal{E} \times[\hat{\theta}, 0]$ under $\mathbf{T}$ from (10). The invariance condition inside the terminal region is given by

$$
\nabla \tilde{E}(y) \cdot \tilde{f}(y, w)+\tilde{F}(y, w) \leq 0 .
$$

Step 2: To map this condition back to the original coordinates $x, \theta, u, v$ we use

$$
\frac{\partial \tilde{E}(y)}{\partial y}=\nabla E(x, \theta) \cdot\left(\begin{array}{cc}
\mathbf{I}^{n \times n} & \frac{\partial p}{\partial \theta} \\
\mathbf{0}^{1 \times n} & 1
\end{array}\right) .
$$

If we apply this relation the invariance condition (9) follows immediately.
In fact, Theorem 1 directly guarantees the main parts of the path-following problem: Path convergence (P1) and constraint satisfaction (P3). Furthermore, assumption A7 requires the timing law $\dot{\theta}=g(\theta, v)$ to be chosen such that $\dot{\theta}>0$ is positive for all admissible $v \in \mathcal{V}$ and all $\theta \in[\hat{\theta}, 0)$. Hence the forward motion of the system along the path is also ensured (P2).

\section{CALCULATION OF STABILIZING END PENALTIES}

In general, the calculation of suitable terminal penalties $E(x, \theta)$ and terminal regions $\mathcal{E}$ is difficult [6]. Subsequently, we use the fact that the vector fields $f$ and $g$ appear decoupled in (9) to find suitable terminal regions and penalties for the proposed MPFC scheme. The main idea of the following considerations is to use the path $\mathcal{P}$ as terminal region. This means to project the invariance condition (9) onto the virtual state $\theta$. Therefore, the cost to drive the system along the path can be calculated in dependence of $\theta$, if some known inputs $u_{\mathcal{E}}$ and $v_{\mathcal{E}}$ guarantee, that the system follows the path. If such inputs are known we only need to check, that for all $\theta \in[\hat{\theta}, 0]$ the costs to follow the path along converge to zero and are upper bounded along $\theta$.

To this end assume that the cost function $F(\cdot)$ for the MPFC problem is

$$
F(x, \theta, u, v)=\left\|\begin{array}{c}
x-p(\theta) \\
\theta
\end{array}\right\|_{Q}^{2}+\left\|\begin{array}{c}
u-\tilde{u} \\
v-\tilde{v}
\end{array}\right\|_{R}^{2},
$$

where $\|x, \theta\|_{Q}^{2}=x^{T} Q x+\hat{q} \theta^{2}, Q, R$ are strictly positive definite matrices and $\hat{q}>0$. We use the structure of (9) to derive the following corollary to Theorem 1.

\section{Corollary 1 (Stabilizing "Zero" End Penalty for MPFC) Suppose that:}

(i) The terminal region $\mathcal{E}$ is equivalent to the path $\mathcal{P}$.

(ii) For all $\theta \in[\hat{\theta}, 0)$ there exists a pair of admissible inputs $u_{\mathcal{E}}$ and $v_{\mathcal{E}}$ which guarantee, that the system follows the path $\mathcal{P}$ with $\dot{\theta}=g\left(\theta, v_{\mathcal{E}}\right)>0$.

(iii) A positive scalar $\epsilon$ is known, such that for all $\theta \in[\hat{\theta}, 0]$

$$
\epsilon>\frac{\hat{q} \theta^{2}+\left\|u_{v_{\mathcal{E}}-\tilde{v}}^{u_{\mathcal{v}}}\right\|_{R}^{2}}{-g\left(\theta, v_{\mathcal{E}}\right) \cdot \theta} \geq 0 .
$$

(iv) The optimal control problem (5)-(8) has a feasible solution for $t_{0}$.

Then, for the closed-loop given by (1), (5)-(8), the end penalty $E(\theta)=\frac{\epsilon}{2} \theta^{2}$ guarantees, that $x(t)-p(\theta(t))$ converges to zero for $t \rightarrow \infty$.

Proof: The corollary expresses the idea to eliminate the state $x$ from the invariance condition (9) of Theorem 1. Our proof directly follows this idea.

Please note, that the path $\mathcal{P}$ is a compact set, since it is the image set of the closed real interval $[\hat{\theta}, 0]$ under the continuous map $p$ from (3). Start at a point on the path $\mathcal{P}$ and apply admissible inputs $u_{\mathcal{E}}$ and $v_{\mathcal{E}}$. These inputs are designed such that the system follows the trajectory given by $p(\theta(t))$, where $\dot{\theta}=g\left(\theta, v_{\mathcal{E}}\right)$. Then, the cost function $F(\cdot)$ from (12) depends only on the arguments $\theta, u, v$ since on $\mathcal{P}$ it holds 
Preprint of accepted submission to 48th IEEE Conference on Decision and Control '09, Shanghai, China.

that $x=p(\theta)$. This leads to a simplified invariance condition in $\mathcal{E}=\mathcal{P}$

$$
\nabla E(\theta) \cdot g\left(\theta, v_{\mathcal{E}}\right)+\hat{q} \theta^{2}+\left\|{ }_{v_{\mathcal{E}}-\tilde{v}}^{u_{\mathcal{V}}-\tilde{u}}\right\|_{R}^{2} \leq 0 .
$$

To obtain the stability condition (13) choose the end penalty as $E(\theta)=\frac{\epsilon}{2} \theta^{2}$ and rearrange (14).

\section{EXAMPLE: AUTONOMOUS VEHICLE}

To exemplify the proposed MPFC scheme an autonomous vehicle in a fixed coordinate frame is considered

$$
\left(\begin{array}{c}
\dot{x}_{1} \\
\dot{x}_{2} \\
\dot{x}_{3}
\end{array}\right)=\left(\begin{array}{c}
u_{1} \cos \left(x_{3}\right) \\
u_{1} \sin \left(x_{3}\right) \\
u_{1} \tan \left(u_{2}\right)
\end{array}\right) \text {. }
$$

The states $x_{1}$ and $x_{2}$ describe the position in the $x_{1}-x_{2}$ plane. $x_{3}$ is the yaw angle. $u_{1}$ refers to the speed of the vehicle and $u_{2}$ is the steering angle. The system inputs are subject to the constraints $u_{1} \in[0,6]$ and $u_{2} \in[-0.63,0.63]$.

The path $\mathcal{P}$ to be followed is given by

$$
\mathcal{P}=\left\{p(\theta) \in \mathbb{R}^{n}:[\hat{\theta}, 0] \mapsto p(\theta)=\left(\begin{array}{c}
\theta \\
\rho(\theta) \\
\arctan \left(\frac{\partial \rho}{\partial \theta}\right)
\end{array}\right)\right\},
$$

where $\rho(\theta)=-\alpha \log (\gamma /(\beta+|\theta|)) \cdot \sin (\omega \theta)$ and $\hat{\theta}=-30$. The coefficients of $\rho(\theta)$ are given by $\alpha=6, \beta=5, \gamma=$ $20, \omega=0.35$. From the definition of the path (16) it can be deduced, that the vehicle should always follow the curve $(\theta, \rho(\theta))^{T}$ along a tangential direction.

The considered cost function is defined by (12) and the weighting matrices are given by $Q=8$. $\operatorname{diag}\left(10^{4}, 10^{5}, 10^{5}, \frac{1}{16}\right)$ and $R=\operatorname{diag}(10,10,1)$. As mentioned previously the path evolution is a degree of freedom in the controller design. It is chosen as $\dot{\theta}=g(\theta, v)=-\lambda \theta+v$, where $\lambda=-10^{-3}$ and $v \in[0,6]$. The initial condition is $\theta_{0}=-30$. Please note, that the chosen path evolution satisfies assumption A7. Since we want to apply Corollary 1 , the terminal region is chosen to equal the path from (16): $\mathcal{E}=\mathcal{P}$.

To apply Corollary 1 we need to know inputs signals $u_{\mathcal{E}}$ and $v_{\mathcal{E}}$. If the system starts on $\mathcal{P}$ and the path evolution $\dot{\theta}$ is defined by $v_{\mathcal{E}}$, then $p(\theta(t))$ is a reference trajectory. Therefore, if $v_{\mathcal{E}}$ is defined, we need to determine the input $u_{\mathcal{E}}$ that guarantees the tracking of $p(\theta(t))$.

Actually, the considered system (15) is differentially flat and a flat output is given by $y=\left(x_{1}, x_{2}\right)^{T}$ (see [7] for details on differential flatness). Regarding $\left(p_{1}(\theta(t)), p_{2}(\theta(t))\right)^{T}$ as a reference trajectory for the considered flat output of (15), it is straightforward to use the flatness property to derive an input which tracks $p(\theta(t))$

$$
\left(\begin{array}{l}
u_{1} \\
u_{2}
\end{array}\right)=\left(\begin{array}{c}
\dot{\theta} \cdot \sqrt{1+\left(\frac{\partial \rho}{\partial \theta}\right)^{2}} \\
\arctan \left(\left(1+\left(\frac{\partial \rho}{\partial \theta}\right)^{2}\right)^{-\frac{3}{2}} \cdot \frac{\partial^{2} \rho}{\partial \theta^{2}}\right)
\end{array}\right) .
$$

This input signal depends on the path parametrization $p(\theta)$ and the parameter evolution specified by $\dot{\theta}=g(\theta, v)$. For $v_{\mathcal{E}}=0$ the input (17) is admissible. Also note, that for $v_{\mathcal{E}}=0$ the second input does not vanish at the end of the path, the reference value for $u_{2}$ is set to $\tilde{u}_{2}=\left.u_{2}\right|_{\theta=0}$ and $\tilde{u}_{1}=0$. It can be verified, that for $\theta \rightarrow 0$ the cost on the path converges faster to zero than the second order polynomial $-\frac{1}{\epsilon} \nabla E \cdot g$. This is equivalent to

$$
\lim _{\theta \rightarrow 0} \frac{\hat{q} \theta^{2}+\left\|\begin{array}{l}
u_{1}-\tilde{u}_{1} \\
u_{2}-\tilde{u}_{2}
\end{array}\right\|_{R}^{2}}{10^{-3} \cdot \theta^{2}}=0 .
$$

Investigating the simplified invariance condition (14) for different values of $\epsilon>0$ shows, that for $\epsilon=1740$ the conditions of Corollary 1 are fulfilled.

Figure 1 shows simulation results for the considered autonomous vehicle (1). The results are obtained using the software package OPTCON [11] and Matlab. Plot a) shows the vehicle movement in the $x_{1}-x_{2}$ plane.

The solid black curve depicts the path-following result, the dashed black curve the result for trajectory tracking for $\dot{\theta}=$ const. $=4.1$. The solid gray line shows the projection of the reference to the $x_{1}-x_{2}$ plane. Both approaches make the vehicle reach the path. Due to the input constraint on $u_{1}$ the tracking approach fails in the last turn, since the reference trajectory is not feasible under the given input constraints. Plot b) shows the input signals, while c) and d) illustrate state signals for both path-following and tracking. Please note, that all system inputs $u_{1,2}$ and the virtual path parameter input $v$ are obtained by repeated optimizations during the application of the proposed MPFC scheme. Comparing the reference evolution for tracking and path-following shows, that the MPFC controller first slows down the reference evolution $(0 \leq t \leq 2$, plot c) $)$ and then speeds it up for $t>2$. Finally, the vehicle reaches at the origin.

\section{CORRIDOR PATH-FOLLOWING}

The main idea of path-following is to relax the timing constraint on a given path. From a practical point of view, however, exact path-following is often not required if the system moves through a specified corridor of the state space, which contains the path. Application examples for the extended setup are keeping a vehicle on track with a given width or flying a plane in a specified air corridor. Subsequently, we will outline how additional spatial degrees of freedom can be implemented into an extension of the proposed MPFC setup. First, we introduce the concept and formal definition of the corridor path-following problem. Second, we show that the MPFC scheme is applicable to this extended problem. Sufficient stability conditions are also presented. Finally, we briefly discuss an example.

So far, the a priori known path is defined as a curve in the state space $\mathcal{P}=\left\{p(\theta) \in \mathbb{R}^{n}: \theta \in[\hat{\theta}, 0] \mapsto p(\theta)\right\}$, see (3). To take additional spatial degrees of freedom into account this formulation is extended to

$$
\mathcal{P}_{k}=\left\{p_{k}(\Theta) \in \mathbb{R}^{n}: \Theta \in[\underline{\Theta}, \bar{\Theta}] \subset \mathbb{R}^{k} \mapsto p_{k}(\Theta)\right\} .
$$

Here $\mathcal{P}_{k}$ is the set of admissible paths. It is given by the map $p_{k}: \Theta \in \mathbb{R}^{k} \mapsto p_{k}(\Theta) \in \mathbb{R}^{n}, k<n$, where $\Theta=$ $\left(\theta_{1}, \ldots, \theta_{k}\right)^{T} \in \mathbb{R}^{k}$ is the vector of the path parameters. The domain of $\Theta$ is the vector valued interval $[\underline{\Theta}, \bar{\Theta}]$. For the first component of $\Theta$ the interval $\theta_{1} \in\left[\hat{\theta}_{1}, 0\right]$ is given. $\theta_{1}$ specifies the direction and orientation of the objective system 
a) Movement in $x_{1}-x_{2}$ Plane

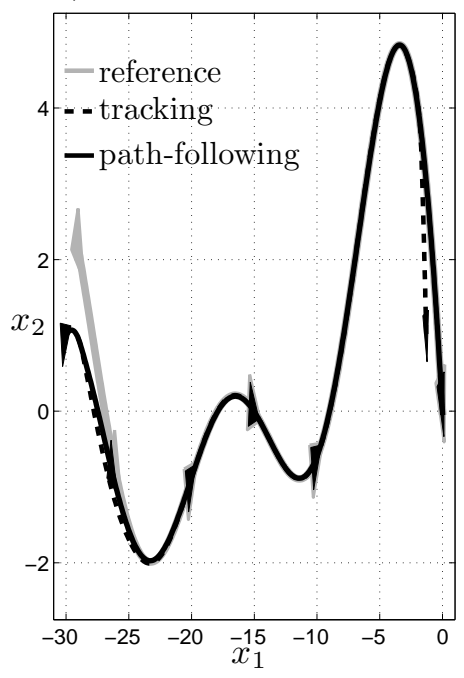

b) Input Signals
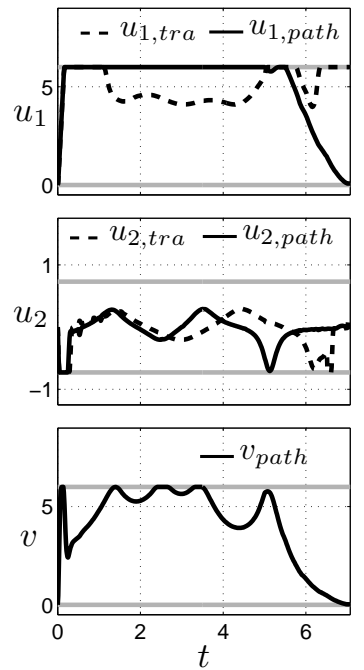

c) Path-Following
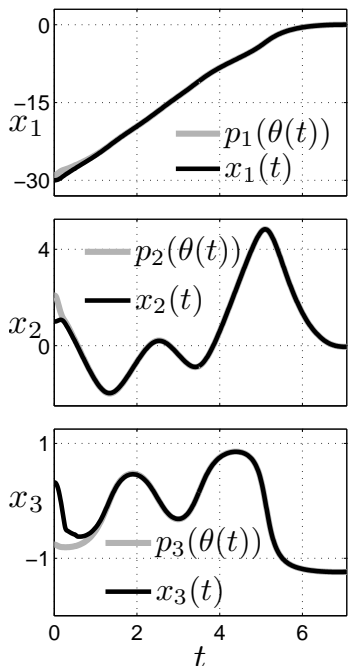

d) Tracking
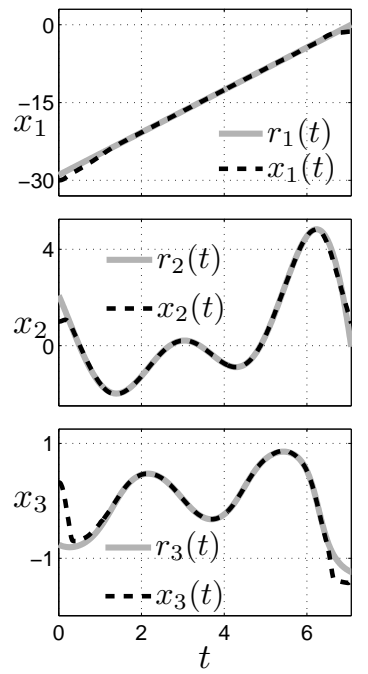

Fig. 1. Simulation results: Application of the MPFC scheme to an autonomous vehicle.

movement in the path corridor $\mathcal{P}_{k}$. The other components $\underline{\theta}_{i} \leq \theta_{i} \leq \bar{\theta}_{i}, \quad i=2 \ldots k$ describe the additional spatial degrees of freedom in $\mathcal{P}_{k}$. The endpoint is $p_{k}(\Theta=0)=0$. Similar to the $1 \mathrm{~d}$ case discussed previously, the map $p_{k}$ is bijective and sufficiently often continuously differentiable. The set $\mathcal{P}_{k}$ is a $k$-dimensional surface in the state space $\mathbb{R}^{n}$.

The main idea of the extended setup is, that the system should move first into the path region $\mathcal{P}_{k}$ and second inside $\mathcal{P}_{k}$ in a specified direction given by $\theta_{1}$ to the origin. In contrast to the $1 \mathrm{~d}$ path-following both the timing and the state space geometry of the reference are not completely specified a priori. The reference in the state space can be influenced by varying the additional parameters $\theta_{2} \ldots \theta_{k}$.

\section{Corridor Path-Following Problem}

Given a system subject to input and state constraints (1), design a controller to satisfy the following:

E1 Path convergence: The system movement through the state space converges to the path corridor $\mathcal{P}_{k}$. Hence $\lim _{t \rightarrow \infty} x(t)-p_{k}(\Theta(t))=0$. Furthermore, the end of the path lies in the origin: $\forall i=1 \ldots k: \lim _{t \rightarrow \infty} \theta_{i}=0$.

E2 Forward motion: $\quad \dot{\theta}_{1}(t)>0$ holds for all $t>0$ and all $\theta_{1} \in\left[\hat{\theta}_{1}, 0\right)$.

E3 Constraint satisfaction: The state and input constraints $x(t) \in \mathcal{X} \subseteq \mathbb{R}^{n}, \quad u(t) \in \mathcal{U} \subset \mathbb{R}^{m}$ are respected for all $t \geq 0$.

The MPFC setup proposed earlier in Section III can be used to solve the extended setup. Similarly to the 1d pathfollowing problem the vector $\Theta$ is used as virtual state in the sketched MPFC formulation.

Instead of one additional state and input this leads to $k$ virtual states $\Theta=\left(\theta_{1} \ldots \theta_{k}\right)^{T}$. The evolution of these states is described by an ODE $\dot{\Theta}=g(\Theta, v)$, where the vector field is given by $g: \mathbb{R}^{k} \times \mathbb{R}^{k} \mapsto \mathbb{R}^{k}$. In fact, $g$ should be chosen such that $\Theta$ is controllable by $v$.

We derive sufficient stability conditions for the proposed MPFC scheme in the same kind and manner as in Theorem 1. Assume A1-A4 are as stated in Section III. Furthermore, the subsequently stated modified assumptions are made:

A5* The cost function $F: \mathcal{X} \times \mathcal{U} \times[\underline{\Theta}, \bar{\Theta}] \times \mathcal{V} \mapsto \mathbb{R}$ is continuous and positive definite in the domain $\mathcal{X} \times \mathcal{U} \times$ $[\underline{\Theta}, \bar{\Theta}] \times \mathcal{V}$.

A6* The path $\mathcal{P}_{k}$ is given by a sufficiently often continuously differentiable, regular, negatively parametrized and bijective map $p_{k}$, such that $\forall \Theta \in[\underline{\Theta}, \bar{\Theta}]: \Theta \mapsto p_{k}(\Theta) \in$ $\mathcal{X} \subseteq \mathbb{R}^{n}$ and $p_{k}(0)=0$ hold.

A7* $g(\Theta, v): \mathbb{R}^{k} \times \mathbb{R}^{k} \mapsto \mathbb{R}^{k}$ has equivalent properties as required for $f(x, u)$ in assumptions A3 and A4. Furthermore, $\forall v \in \mathcal{V}$ and $\forall \theta_{1} \in\left[\hat{\theta}_{1}, 0\right): \dot{\theta}_{1}>0$, where $\mathcal{V} \subset \mathbb{R}^{k}$ is compact and $0 \in \mathcal{V}$.

The following theorem holds:

\section{Theorem 2}

Consider the extended path-following problem for (1) as given by E1-E3 and assume that assumptions A1-A4 and $A 5^{*}-A 7^{*}$ are fulfilled. Suppose that:

(i) The terminal region $\mathcal{E}$ is compact and $\mathcal{P}_{k} \subseteq \mathcal{E}$. The terminal penalty $E(x, \Theta)$ is continuously differentiable, positive semidefinite and $E(0,0)=0$ holds.

(ii) For all $(x, \Theta) \in \mathcal{E} \times[\underline{\Theta}, \bar{\Theta}]$ there exists a pair of admissible inputs $\left(u_{\mathcal{E}}, v_{\mathcal{E}}\right) \in \mathcal{U} \times \mathcal{V}$ such that

$$
\nabla E(x, \Theta) \cdot\left(\begin{array}{l}
f\left(x, u_{\mathcal{E}}\right) \\
g\left(\Theta, v_{\mathcal{E}}\right)
\end{array}\right)+F\left(x, \Theta, u_{\mathcal{E}}, v_{\mathcal{E}}\right) \leq 0
$$

and the solutions of $\dot{x}=f\left(x, u_{\mathcal{E}}\right)$ starting at $x \in \mathcal{E}$ stay in $\mathcal{E}$, while the solutions of $\dot{\Theta}=g\left(\Theta, v_{\mathcal{E}}\right)$ starting at $\Theta \in[\underline{\Theta}, \bar{\Theta}]$ stay in $[\underline{\Theta}, \bar{\Theta}]$ for all times and $\lim _{t \rightarrow \infty} \Theta(t)=$ 0 . 
(iii) The NMPC open-loop optimal control problem is feasible for $t_{0}$.

Then, for the closed-loop system defined by (1), (5)-(8), the path-following error $e_{p}(t)=x(t)-p_{k}(\Theta(t))$ converges to zero for $t \rightarrow \infty$. Furthermore the region of attraction is given by the set of states for which the open-loop optimal control problem has a feasible solution.

The proof follows the one of Theorem 1and is thus omitted. In fact, the crucial steps in the proof of Theorem 1 do not explicitly rely on the fact that the path was considered to be 1-dimensional only. Please note, that the path corridor $\mathcal{P}_{k}$ is not a constraint for the state. Only the reference is restricted to lie within the corridor $p_{k}(\Theta(t)) \in \mathcal{P}_{k}$.

\section{EXAMPLE}

Consider the autonomous vehicle from Section IV. For this system a corridor path-following problem can be defined. The new control objective is not to converge to the path $\mathcal{P}$ given by (16) but to converge to a region in the neighborhood of the path. More formally, the vehicle movement should converge to a path corridor $\mathcal{P}_{2}=\left\{q(\Theta) \in \mathbb{R}^{3}: \Theta \mapsto q(\Theta)\right\}$ which is given by

$$
q(\Theta)=p\left(\theta_{1}\right)+\left(\begin{array}{c}
-\theta_{2} \cdot \frac{\partial \rho}{\partial \theta_{1}}\left\|\left(\theta_{1}, \rho\left(\theta_{1}\right)\right)\right\|^{-1} \\
\theta_{2} \cdot\left\|\left(\theta_{1}, \rho\left(\theta_{1}\right)\right)\right\|^{-1} \\
\arctan \left(\frac{\partial \theta_{2}}{\partial \theta_{1}}\right)
\end{array}\right) .
$$

The path parameter domain is given by $\Theta=\left(\theta_{1}, \theta_{2}\right)^{T} \in$ $[-30,0] \times[-1.25,1.25] \subset \mathbb{R}^{2}$. As in the previous example the path parameters dynamics are chosen as linear ODEs $\dot{\theta}_{1}=-10^{-3} \theta_{1}+v_{1}$ and $\dot{\theta}_{2}=-10^{-2} \theta_{2}+v_{2}$, where the virtual inputs $v_{1}$ and $v_{2}$ belong to $v_{1} \in[0,6], v_{2} \in[-5,5]$.

Figure 2 shows the simulation results for this example. The black dash-dot lines mark the projection of $\mathcal{P}_{2}$ into the $x_{1}-x_{2}$ plane and the dashed gray line depicts the projection of $\mathcal{P}$ from (16) into the $x_{1}-x_{2}$ plane. The solid Grey line shows the reference $q(\Theta(t))$ calculated by the controller in the $x_{1}-x_{2}$ plane. The dashed black line depicts the system movement, if the proposed MPFC scheme is applied to the extended pathfollowing problem. The MPFC controller drives the vehicle to the set $\mathcal{P}_{2}$. Inside the set it follows the reference $q(\Theta(t))$, which is specified by the repeated online optimizations of the MPFC scheme.

\section{CONCLUSIONS AND OUTLOOK}

In this contribution we propose a model predictive control approach for different nonlinear path-following problems. Our control scheme explicitly considers input and state constraints while guaranteeing stability. Novel stability results -based on the idea to use the path as terminal region- for the usual 1d path-following problem have been presented. Furthermore, a corridor path-following problem considering additional spatial degrees of freedom in the path description has been introduced. We show that the proposed MPFC setup is also suitable for the extended problem. Future works will focus on inherent robustness properties of MPFC and the calculation of tubes as terminal regions.

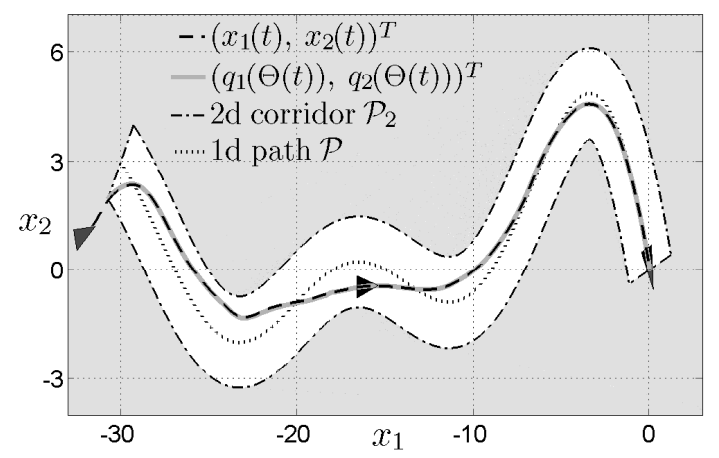

Fig. 2. Simulation results for $2 \mathrm{~d}$ corridor path-following.

\section{ACKNOWLEDGMENTS}

The authors gratefully acknowledge the very helpful discussions with Friedrich von Haeseler. Furthermore, we thank the government of Saxony-Anhalt for funding this work under grant 0904/00067.

\section{REFERENCES}

[1] A. P. Aguiar, J. P. Hespanha, and P. V. Kokotovic. Path-following for nonminimum phase systems removes performance limitations. IEEE Trans. Automat. Contr., 50(2):234-239, 2005.

[2] A. P. Aguiar, J. P. Hespanha, and P. V. Kokotovic. Performance limitations in reference tracking and path following for nonlinear systems. Automatica, 44(3):598-610, 2008.

[3] A. Bemporad. Reference governor for constrained nonlinear systems. IEEE Trans. Automat. Contr., 43(3):415-419, 1998.

[4] K. D. Do and J. Pan. Global robust adaptive path following of underactuated ships. Automatica, 42(10):1713-1722, 2006.

[5] T. Faulwasser and R. Findeisen. Assessment and future directions of nonlinear model predictive control, chapter Nonlinear model predictive path-following control, pages 335-343. Lecture Notes in Control and Information Sciences, Springer Heidelberg/Berlin, 2009.

[6] R. Findeisen. Nonlinear Model Predictive Control: A Sampled-Data Feedback Perspective. Fortschr.-Ber. VDI Reihe 8 Nr. 1087, VDI Verlag, 2006.

[7] M. Fliess, J. Lévine, P. Martin, and P. Rouchon. Flatness and defect of non-linear systems: introductory theory and examples. Int. J. Contr., 61(6):1327-1361, 1995.

[8] F. Fontes. A general framework to design stabilizing nonlinear model predictive controllers. Sys. Contr. Lett., 42(2):127-143, 2001.

[9] D. Q. Mayne, J. B. Rawlings, C. V. Rao, and P. O. M. Scokaert. Constrained model predictive control: Stability and optimality. Automatica, 36(6):789-814, 2000.

[10] H. Michalska. Trajectory tracking control using the receding horizon strategy. In Symposium on Control, Optimization and Supervision: CESA '96 IMACS, 1996.

[11] Z. K. Nagy. Model based control approach for batch crytallization product design. In Proc. 17th IFAC World Congress, 2008.

[12] J.A. Rossiter and B. Kouvaritakis. Reference governors and predictive control. In Proc. American Control Conference 1998, volume 6, pages 3692-3693, 1998.

[13] R. Skjetne, T. Fossen, and P. Kokotovic. Robust output maneuvering for a class of nonlinear systems. Automatica, 40(3):373-383, 2004. 